\title{
Vascular Endothelial Growth Factor in Gingival Crevicular Fluid Around Dental Implants
}

\author{
E. Mierzwinska-Nastalska, L. Lomzynski, M. Jaworska-Zaremba, J. Kostrzewa-Janicka \\ Department of Prosthodontics, Warsaw Medical University, Warsaw, Poland
}

\begin{abstract}
Introduction: Angiogenesis occurs under physiological and pathological conditions and is regulated by cytokines and growth factors. Vascular endothelial growth factor (VEGF) is a multifunctional angiogenic cytokine that plays a significant role in inflammation and immune responses implicated in the pathogenesis of inflammatory processes in the implant- surrounding tissues.

Objective: The study investigated the concentration of VEGF in gingival crevicular fluid (GCF) in healthy and diseased soft tissues surrounding implants.

Material and methods: Clinical examinations were focused on assessing the periodontal status of soft tissues around dental implants with the use of Florida Probe. Bone loss was examined radiologically. VEGF concentrations were assessed by enzyme-linked immunoabsorbent assay (ELISA).

Results: VEGF concentrations were found higher in crevicular fluid around implants than in clinically healthy sites. They were also strongly correlated with the pocket depth.

Conclusions: The presence of VEGF in gingival crevicular fluid in patients with peri-implants can be implicated in the progression of peri-implantitis, possibly by promoting the formation of new blood vessels during angiogenic processes.
\end{abstract}

Key words: dental implants, blood vessels, peri-implants, vascular endothelial growth factor

\section{INTRODUCTION}

In patients under the implant-prosthetic treatment with the use of endosseous implants, the occurrence of osseointegration, regeneration and healing processes is observed in tissues around implants. However, like in the periodontium of the patient's own teeth, inflammatory processes in peri-implant tissues, termed peri-implantitis, may develop [1]. The inflammatory process is confined to soft tissues surrounding the implant and occurs along with progressive peri-implant bone loss. It is a chronic process with episodes of aggravation and remission. Esposito et al [2] have defined peri-implantitis as a site-specific, plaque-induced infection with progressive loss of the bone supporting a functioning implant. Pocket formation, bone de- struction, bleeding at gentle probing, swelling, tissue color changing, erythema, and frequent suppuration are recognized as the most common signs of inflammation.

The appearance of inflammatory infiltration in implant-surrounding tissues accompanied by the progression of the whole process may be the cause of a failing implant. In the available literature, various possible causes have been listed, but there is no clear-cut answer on what biological mechanisms are responsible for these lesions. A significant role in the destruction and healing processes is attributed to the process of angiogenesis; however. Its role in the development of inflammation or healing of periodontal tissues has not as yet been clarified [3, 4].

The process of angiogenesis is strictly controlled and depends on the coordination and production of angiogenic agents, which play the role in both stimulation and inhibition. Although interleukins (IL-1, IL-6, and IL-8), growth factors (transforming and epidermal), fibroblast growth factor (FGF), and tumor necrosis factor (TNF) are most frequently mentioned among various agents that influence angiogenesis, the vascular endothelial growth factor (VEGF) is the strongest and most specific endogenic stimulus of angiogenesis [5, 6]. Monocytes and macrophages present in infiltration are, like vascular endothelial cells, able to produce angiogenesis-generating factors, such as VEGF [3].

VEGF performs the following numerous functions: stimulates proliferation of vascular endothelial cells, activates the excretion of proteolytic enzymes, and intensifies chemotaxis and migration. All the aforesaid processes are essential for angiogenesis. In addition, VEGF is a peptide that induces vascular endothelium permeability for fluids and proteins and is able to bind endothelial cells [7].

Bacterial antigens as well as inflammation mediators and products are risk markers that contribute to the induction of active inflammatory process. Prostaglandin $\mathrm{PGE}_{2}$ is especially linked with an active destruction of epithelial attachment and what is more important, it is a strong stimulus of VEGF synthesis [8]. In addition, VEGF induction is influenced by IL1, IL-6, TNF, and hypoxia. VEGF growth occurs especially as a response to specific bacterial risk factors. All these factors may stimulate VEGF excretion that 
directly influences the process of angiogenesis. This process may in turn contribute to the development of inflammation by affecting the ability of new blood vessels to transport pro-inflammatory cells, nutrients, and oxygen to inflammatory tissues $[5,6]$.

The aim of the present study was to assess the expression of vascular endothelial growth factor in gingival crevicular fluid around implants in patients after the prosthetic-implant treatment.

\section{Material AND Methods}

The study was performed in accordance with the Declaration of Helsinki for Human Research and study protocol was approved by the Ethics Committee of Warsaw Medical University in Warsaw, Poland.

Thirty patients (13 males and 17 females, aged 35-82; mean 58.5 years) with partial or complete loss of teeth, under the prosthetic treatment with the use of endosseous implants, and provided with fixed or movable dentures, were eligible for the study. These patients had the implant procedure performed at least one year earlier. The study material comprised gingival crevicular fluid around implants with implant-surrounding soft tissues, healthy or changed by the inflammatory process. The following criteria were applied to select the peri-implantitis patients (the study group): the presence of bleeding on probing, peri-implant pockets of more than $3 \mathrm{~mm}$ deep, radiological evidence of peri-implant bone loss, swelling and gingival erythema around the implant. The control group was composed of the patients with healthy peri-implant tissues, probing depth of no more than $2 \mathrm{~mm}$, no bleeding on probing, no gingival erythema or accumulation of bacterial plaques. Patients with diabetes, rheumatoid arthritis, coronary heart disease, and smokers were excluded from the study. In all cases, there was clinical and radiographic evidence of osseointegration signs confirmed by the results of the implant stability examination performed with use of Ostell and Periotest devices.

On clinical examination, in addition to visual observations, plaque index, bleeding, and pocket depth around implants were assessed with the use of the Florida Probe (Florida Probe Corporation, USA). On radiological examination, the extent of peri-implant bone loss was assessed with the right-angle method. To achieve the major aim of the study, laboratory tests were carried out to assess the concentration of VEGF in gingival crevicular fluid around implants by employing enzyme-linked immunoabsorbent assay (ELISA). The tests were performed with diagnostic kits (Pharmingen, USA) in the Department of Laboratory Diagnostics of the Institute National of Tuberculosis and Lung Diseases in Warsaw, Poland.

The samples of gingival crevicular fluid $(0.3 \mathrm{ml}$ each) were collected into Eppendorf tubes, using a sample syringe with a blunt needle, diluted in phosphate-buffered saline (PBS), incubated, and finally centrifuged. In the ELISA test, the absorbance was read in a microplate spectrophotometer with the use of ready-made plates. In the study material, VEGF activity was determined relative to the calibrator and expressed as total VEGF in $\mathrm{pg} / \mathrm{ml}$.
RESULTS

Table 1. Total vascular endothelial growth factor (VEGF) concentration in gingival crevicular fluid around implants.

\begin{tabular}{lc}
\hline Group & VEGF $(\mathrm{pg} / \mathrm{ml})$ \\
\hline Study group $(\mathrm{n}=39)$ & $86.28 \pm 0.21$ \\
Control group $(\mathrm{n}=20)$ & $25.21 \pm 0.32$ \\
Statistical significance & $\mathrm{P}<0.001$ \\
\hline
\end{tabular}

Table 2. Concentration of vascular endothelial growth factor (VEGF) in gingival crevicular fluid around implants by the depth of pockets.

\begin{tabular}{cccc}
\hline Pocket depth & $\begin{array}{c}\text { Number } \\
\text { of samples }\end{array}$ & $\begin{array}{c}\text { VEGF (pg/ml) } \\
(\bar{x} \pm \mathrm{SE})\end{array}$ & Significance \\
\hline$\leq 2 \mathrm{~mm}$ & 20 & $25.21 \pm 0.32$ & $\mathrm{NS}$ \\
$\leq 3 \mathrm{~mm}$ & 23 & $80.42 \pm 0.98$ & $\mathrm{P}<0.001$ \\
$4-6 \mathrm{~mm}$ & 16 & $92.17 \pm 0.19$ & $\mathrm{P}<0.01$ \\
\hline
\end{tabular}

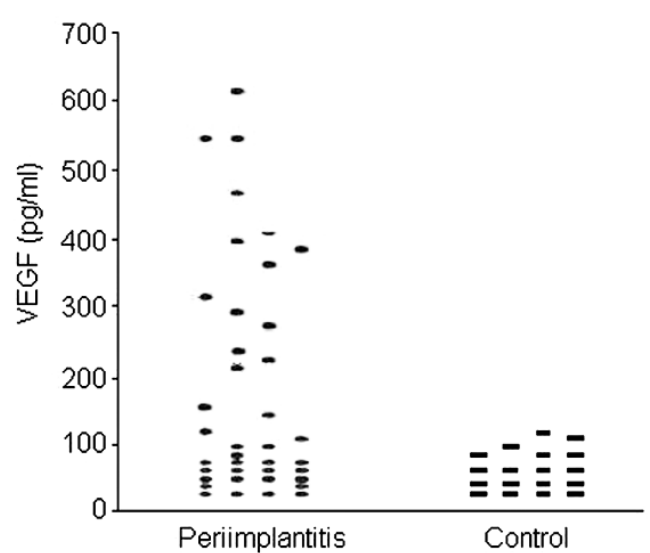

Fig. 1. Concentration of vascular endothelial growth factor (VEGF) (pg/ml) in gingival crevicular fluid around implants.

The results of the study indicated significantly higher concentrations of total VEGF in the study than in the control group of persons with healthy tissues around implants (Table 1; Fig. 1). Having analyzed individual cases, it was found that the difference between the results was more pronounced in the study than in the control group; in the latter lower values were observed. The concentration of VEGF positively correlated with clinical signs of peri-implantitis in patients with deeper periodontal pockets and other signs of inflammation (Table 2).

The results of the study also point to a correlation between VEGF concentration in gingival fluid and the bacterial plaque index (accumulation of plaques on the implant components). In the patients showing more pronounced hygiene negligence, a higher concentration of VEGF was observed (Fig. 2). 


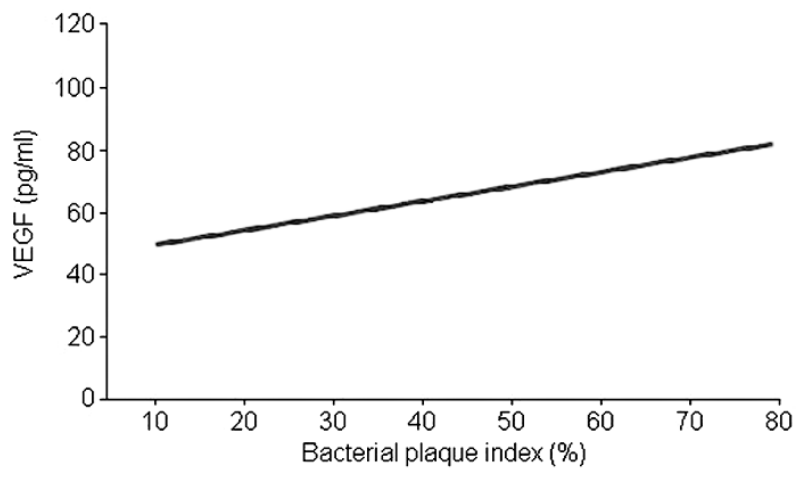

Fig. 2. Correlation between the expression of vascular endothelial growth factor (VEGF) and bacterial plaque index.

\section{Discussion}

Reports published in the available literature have confirmed the relationship between periodontal disease (periodontitis) and inflammation of tissues surrounding the implant (peri-implantitis), which may lead to a failing implant and a failure in the implant-prosthetic treatment $[9,10]$. In the etiopathogenesis and progression of both processes, the presence of gram-positive anaerobic flora, the composition of inflammatory infiltrate, and the expression of inflammatory mediators play an important role $[11,12]$. In soft tissues surrounding the failing implant, a large number of microphages, lymphocytes, leucocytes, and plasmic cells have been found [13].

The enhanced expression of inflammatory mediators promotes the process of angiogenesis. The development of inflammation in teeth-surrounding tissues affects the periodontal vasculature. In an early stage of the disease, the distortion of perivascular connective tissues is observed. This is followed by the destruction of collagen fibers and rapid filling of emerging spaces with inflammatory cells and loose connective tissues. Finally, capillaries proliferate into these spaces during the process of angiogenesis [14]. The network of new blood vessels, produced during this process, may contribute to the enhanced inflammation as a result of a possible transport of pro-inflammatory cells, nutrients, and oxygen by these new cells to the inflamed tissues [15].

The results of the present study indicate the presence of VEGF in both healthy periodontal tissues and in tissues of patients with diagnosed peri-implantitis, which confirms an important role of VEGF in both cases. However, a higher VEGF concentration found in gingival crevicular fluid of patients affected by inflammation around implants provides evidence that VEGF can be regarded as a potential factor that initiates gingivitis and periodontitis by promoting the expansion of the vascular network. Paradis et al [7], evaluating microvessel density (MVD) with the use of a histomorphometric technique, have observed the correlation between VEGF expression and revascularization. Johnson et al [2] have evidenced the increased density of the vascular network correlated with the depth of gingival pockets in patients with periodontal disease. The results obtained in the present study are similar to those demonstrated by the above-cited authors. We here found higher VEGF concentrations in patients with deeper periodontal pockets observed on the clinical examination employing the Florida Probe.

The present study also demonstrates a significant effect of bacterial plaques present in implant components on the VEGF concentration in gingival crevicular fluid. A number of factors may contribute to a failed implant. Mombelli and Lang [10] have found a correlation between the accumulation of bacterial plaques and the severity of inflammation in soft tissues surrounding the implant. The role of VEGF in the healing process of tissues around the implant and in the developing of pathologies requires further exploration.

\section{CONCLUSIONS}

1. More pronounced expression of VEGF in gingival fluid of patients with peri-implantitis may be associated with the process of angiogenesis and expansion of the vascular network.

2. Vascular endothelial growth factor likely factors in the initiation and progression of inflammation of peri-implant tissues through the stimulation of vasculature development in the process of angiogenesis coexisting with inflammation progression.

Conflicts of interest: No conflicts of interests were declared by the authors in relation to this article.

\section{REFERENCES}

1. Karoussis I, Kotsovilis S. A comprehensive and critical review of dental implant prognosis in periodontally compromised partially edentulous patients. Clin Oral Impl Res 2007; 18: 669-79.

2. Johnson RB, Serio FG, Dai X. Vascular endothelial growth factors and progression of periodontal diseases. J Peroidontol 1999; 70: 848-52.

3. Brown LF, Yeo KT, Berese B, Yeo TK, Senger DR, Dvorak HF, van de Water L. Expression of vascular permeability factor (vascular endothelial growth factor) by epidermal keratinocytes during wound healing. J Exp Med 1992; 176: 1375-9.

4. Esposito M, Hirsh J, Lekholm U, Thomsen P. Differential diagnosis and treatment strategies for biologic complications and failing oral implants: A review of the literature. Int J Oral Maxillofac Implants 1999; 14: 473-90.

5. Dvorak HF, Brown LF, Detmar M, Dvorak AM. Vascular permeability factor/vascular endothelial growth factor, microvascular permeability and angiogenesis. Am J Pathol 1995; 146: 1029-39.

6. Leung DW, Cachianes G, Kuang W-J, Goeddel DV, Ferrara N. Vascular endothelial growth factor is a secreted angiogenic mitogen. Science 1989; 246: 1306-9.

7. Paradis V, Lagha NB, Zeimoura L, Blanchet P, Eschwege P, Ba N, Benoit G, Jardin A, Bedossa P. Expression of vascular endothelial growth factor in renal cell carcinomas. Virchows Arch 2000; 436: 351-6.

8. Ben-Av P, Crofford LJ, Wilder RL, Hla T. Induction of vascular endothelial growth factor expression in synovial fibroblasts by prostaglandin $\mathrm{E}$ and interleukin-1: A potential mechanism for inflammatory angiogenesis. FEBS Lett 1995; 372: 83-7.

9. Mombelli A. Etiology, diagnosis, and treatment considerations in peri-implantitis. Curr Opin Periodontol 1997; 4: 127-36. 
10. Mombelli A, Lang NP. The diagnosis and treatment of peri-implantitis. Periodontol 2000. 1998; 17: 63-76.

11. McGee JM, Tucci MA, Edmundson TP, Serio CL, Johnson $\mathrm{RB}$. The relationship between concentrations of proinflammatory cytokines within gingiva and the adjacent sulcular depth. J Periodontol 1998; 69: 865-71.

12. Takahashi K, Lappin D, Kinane DF. In situ localization of cell synthesis and proliferation periodontitis gingiva and tonsillar tissue. Oral Dis 1996; 2: 210-6.

13. Esposito M, Hirsch JM, Lekholm U, Thomsen P. Biological factors contributing to failures of osseointegrated oral implants. II. Etiopathogenesis. Eur J Oral Sci 1998; 106: 721-64.

14. Booth V, Young S, Cruchley A, Taichman NS, Palcolog E. Vascular endothelial growth factor in human periodontal disease. J Periodont Res 1998; 33: 491-9.
15. Roberts FA, McCaffery KA, Michalek SM. Profile of cytokine MRNA expression in chronic adult periodontitis. J Dent Res 1997; 75: 1833-9.

Address for correspondence:

Elzbieta Mierzwinska-Nastalska

Department of Prosthodontics

Warsaw Medical University

59, Nowogrodzka St.

02-006 Warsaw

Poland

Phone: +48 225021886 